\title{
UN CASO DIFÍCIL EN EL CÓDIGO CIVIL ESPAÑOL
}

\section{Introducción: Una aplicación de las teorías de Alchourrón y Bulygin y de MacCormick}

n el ámbito de la filosofía jurídica, los modelos teóricos suelen ser propuestos, criticados, defendidos, refutados, alabados, y hasta defenestrados. En pocas ocasiones son usados.

Esto puede resultar curioso: un modelo teórico pretende describir alguna parte del fenómeno jurídico, y una de las maneras de demostrar su plausibilidad es usándolo, esto es, aplicarlo a un caso jurídico concreto.

En este sentido el presente trabajo puede ser calificado más de práctico que de teórico, ya que de lo que se trata es de usar modelos teóricos. En concreto usaré dos modelos: el del análisis lógico de Carlos Alchourrón y Eugenio Bulygin (A \& B, en adelante) y el de la argumentación jurídica de Neil MacCormick.

A través de un problema jurídico concreto (arts. 453 a 455 del código civil español) intentaré demostrar la utilidad de la lógica para la sistematización del derecho (y con esto la plausibilidad del modelo de A \& B) y la utilidad y los límites de la argumentación jurídica en la tarea modificadora del derecho (y con esto la plausibilidad del modelo de MacCormick). En concreto, el modelo de A \& B se usará para detectar una laguna normativa, y el de MacCormick para completar el sistema.

En 1971 A \& B publican un interesante trabajo titulado «Normative Systems» (ver A \& B 1971), en el que proponen un modelo lógico con reglas de inferencia aplicables a los sistemas normativos. Los autores detectan una laguna en el código civil argentino, y examinan las opiniones de distintos dogmáticos.

El presente seguirá esta línea de trabajo, sin criticar ni modificar el modelo; sino solo usándolo. 
Sin embargo, a diferencia de A \& B 1971, este trabajo propondrá una manera de completar el sistema, basándose en el modelo de MacCormick, en concreto su tesis acerca de la coherencia normativa de los sistemas jurídicos (ver MacCormick 1978).

El objetivo del presente es resaltar algunos problemas que puede tener la dogmática jurídica al analizar un caso. Sostendré que el éxito depende de distinguir claramente los distintos tipos de problemas, y afrontar cada uno con métodos distintos.

Para A \& B la dogmática jurídica es una actividad compleja en la que pueden y deben distinguirse por lo menos tres etapas distintas: 1) la identificación de las normas jurídicas; 2) la sistematización de tales normas; y 3) la modificación o transformación de los sistemas jurídicos ${ }^{1}$.

En el caso concreto que analizaré, la primera de las tareas no resultará compleja, ya que las normas del sistema no plantean graves problemas de interpretación, puesto que su sentido puede precisarse por medio de la interpretación semántica.

Las tareas 2) y 3) ocuparán el centro del trabajo. A lo largo del mismo sostendré que el modelo lógico-analítico y el de la argumentación jurídica son dos modelos distintos porque se ocupan de problemas distintos: El problema de la sistematización del derecho es de dominio exclusivo de la lógica; y el problema de la modificación o transformación del derecho es de dominio compartido de la lógica y de la teoría de la argumentación jurídica. Sostendré, además, que la lógica es una herramienta importante para distinguir entre casos fáciles y casos difíciles.

\section{CAPITULO PRIMERO: IDENTIFICACIÓN Y SISTEMATIZACIÓN}

\section{Aproximación al sistema normativo: El universo del discurso}

Nuestro caso jurídico es una cuestión del código civil español, en concreto, el reintegro de los gastos y mejoras en la liquidación de situaciones posesorias.

Para ilustrarlo expondré un ejemplo. Juan, poseedor de un bien (un inmueble, por ejemplo) ha sido vencido en juicio por Pedro (el propietario del bien, por ejemplo), y debe traspasarle la posesión por sentencia. El poseedor vencido, Juan, ha realizado determinados gastos y mejoras (por ejemplo: pago de impuestos territoriales, pintó la casa, cambió las cañerías) durante el tiempo que duró su posesión, y

${ }^{1}$ Bulygin 1986; y Alchourrón 1986. 
requiere una compensación por dichas erogaciones. ¿En qué circunstancias, el poseedor vencido, Juan, tiene derecho a ser compensado por esas erogaciones? Dicho de otra manera: ¿En qué circunstancias, el vencedor y nuevo poseedor, Pedro, está obligado a compensar los gastos y las mejoras realizadas por el anterior poseedor?

Esta situación constituye nuestro Universo del Discurso ${ }^{2}$ (UD en adelante). El UD delimita el ámbito del análisis, ya que sólo nos ocuparemos de aquellos estados de cosas que pueden ser encuadrados como dentro de nuestro UD.

En nuestro caso, el UD consiste en tratarse de un gasto o mejora realizado por un poseedor, que ha sido vencido en la posesión. Podemos agregar, en cuanto a las mejoras, que deben tratarse de mejoras existentes al momento en que se traspasa la posesión y de mejoras no provenientes de la naturaleza y del tiempo (aunque esto último es redundante, ya que nos referimos a «gasto o mejora realizada por el poseedor vencido»). Todo esto en virtud de los artículos 456 y $458^{3}$ que eliminan del régimen de compensación estos dos tipos de mejoras.

De esta forma, la propiedad definitoria de nuestro UD es la siguiente: Ser un gasto o mejora existente al momento del traspaso de la posesión, realizado por un poseedor que ha sido vencido en su posesión.

Todo elemento del UD es un estado de cosas o situación en el cual un poseedor que ha realizado gastos y mejoras es vencido en su posesión. El UD es el conjunto de todos esos estados de cosas y situaciones, y quedan fuera de nuestro análisis aquellas situaciones que no se refieren a nuestro $\mathrm{UD}^{4}$.

\section{El ámbito fáctico del problema: El universo de casos}

Delimitado el UD, nuestra pregunta es la siguiente: ¿En qué circunstancias el nuevo poseedor está obligado a abonarle al anterior poseedor los gastos y mejoras realizados?

\footnotetext{
${ }^{2}$ Sobre la noción de Universo del Discurso ver A \& B 1971.

${ }^{3}$ Art. 456 (del Código Civil Español): «Las mejoras provenientes de la naturaleza o del tiempo ceden siempre en beneficio del que haya vencido en la posesión».

Art. 458: «El que obtenga la posesión no está obligado a abonar mejoras que hayan dejado de existir al adquirir la cosa».

${ }^{4}$ Se han utilizado los arts. 456 y 458 para delimitar el UD, porque nos interesan los problemas que plantean los arts. 453 a 455 del Código Civil. Esto no significa que no sea posible ampliar el UD y utilizar los arts. 456 y 458 como axiomas del sistema. Esta segunda opción ampliaría los casos del Universo de Casos (UC) a 12 ó 24, según la interpretación que se realice de los mismos, lo que complicaría el uso de la matriz.
} 
La respuesta depende de las circunstancias que el legislador ha tenido en cuenta. De los arts. 453 a 455 se desprenden dos tipos de circunstancias: La buena o mala fe del anterior poseedor y el tipo de gasto o mejora que se ha realizado.

A partir de estos dos tipos de circunstancias (o propiedades fácticas) se construirá el Universo de Casos (UC). El UC es el conjunto de todos los casos mínimos posibles.

Este conjunto debe ser una división, esto es que los casos del UC deben ser conjuntamente exhaustivos, mutuamente excluyentes y lógicamente no vacios ${ }^{5}$. Los casos del UC son casos elementales, esto es, casos mínimos, ya que no pueden ser subdivididos en otros sub-casos ${ }^{6}$.

Para la construcción de nuestro UC se analizará cada tipo de circunstancia por separado.

En relación al tipo de gasto o mejora, el legislador divide entre necesarios, útiles y suntuarios. Como señala La Cruz Berdejo 1988, esta clasificación (como muchas otras) plantea problemas de vaguedad, ya que un mismo objeto, por ejemplo, una jaula para leones, puede ser un lujo para quien piensa decorar su parque; un gasto útil para un zoológico y un gasto necesario para quien empieza por tener a su lado al león ${ }^{7}$.

De esta forma pueden aparecer problemas a la hora de calificar determinados gastos o mejoras realizados por el anterior poseedor.

Sin embargo, esta cuestión no importa problemas lógicos. La clasificación en gastos necesarios, útiles y suntuarios conforman una división, ya que dichas propiedades son excluyentes entre sí (si es un gasto necesario no es útil ni suntuario, etc.) y exhaustivas del UD (todo gasto es o necesario, o útil o suntuario). El UC de estas propiedades puede configurarse de la siguiente manera:

\begin{tabular}{ccccc}
\hline \multicolumn{5}{c}{ Gráfico 1 } \\
\hline & GN & GU & GS \\
$1)$ & + & - & - & GN \\
$2)$ & - & + & - & GU \\
$3)$ & - & - & + & GS \\
\hline
\end{tabular}

${ }^{5}$ A \& B 1971, págs. 56 y 57.

${ }^{6}$ Sobre la noción de caso elemental ver A \& B 1971, pág. 35.

${ }^{7}$ El ejemplo está tomado de La Cruz Berdejo 1988, pág. 116. 
El caso 1) es el del gasto necesario (no útil, no suntuario), el 2) el del gasto útil (no necesario, no suntuario), y el 3) el del gasto suntuario (no necesario, no útil ${ }^{8}$.

La otra circunstancia que el legislador considera relevante se refiere a la fe del anterior poseedor. Existen dos posibilidades solamente: buena fe o ausencia de buena fe (mala fe). La división entre buena y mala fe es exhaustiva y excluyente: Todos los poseedores o son de buena fe o son de mala fe; si un poseedor es de buena fe no puede ser calificado también como de mala fe; y viceversa. El UC de esta propiedad puede ser representado del siguiente modo:

\section{Gráfico 2}

$\mathrm{BF}$

$$
\begin{aligned}
& \text { 1) }+ \text { BF (Buena fe) } \\
& \text { 2) }-\neg \text { BF (No buena fe: mala fe) }
\end{aligned}
$$

Combinando los tipos de gastos o mejoras (3 casos - gráfico 2) con los tipos de poseedor (2 casos - gráfico 3), obtenemos un UC de 6 casos $(3 \times 2=6)$.

\section{Gráfico 3}

\begin{tabular}{lcccccccc}
\hline & GN & GU & GS & BF & & & \\
$1)$ & + & - & - & + & $\mathrm{GN}$ & $\wedge$ & $\mathrm{BF}$ \\
$2)$ & + & - & - & - & $\mathrm{GN}$ & $\wedge$ & $\neg \mathrm{BF}$ \\
$3)$ & - & + & - & + & $\mathrm{GU}$ & $\wedge$ & $\mathrm{BF}$ \\
$4)$ & - & + & - & - & $\mathrm{GU}$ & $\wedge$ & $\neg \mathrm{BF}$ \\
$5)$ & - & - & + & + & $\mathrm{GS}$ & $\wedge$ & $\mathrm{BF}$ \\
$6)$ & - & - & - & - & $\mathrm{GS}$ & $\wedge$ & $\neg \mathrm{BF}$ \\
\hline
\end{tabular}

Cada uno de los casos del UC es un caso elemental. Los casos elementales no pueden ser subdividos porque son casos mínimos: contemplan todas las propiedades o circunstancias relevantes determinadas por el legislador.

${ }^{8}$ Este universo de casos es del mismo tipo que los universos de casos utilizados en los impuestos, donde cada caso es caracterizado por valores numéricos. Ver A \& B 1971, pág. 56. 
Los casos que no son elementales son complejos ${ }^{9}$. Los casos complejos no contemplan todas las circunstancias relevantes, y pueden ser subdivididos en casos elementales. Veremos como la norma 453 (1), a partir de un caso complejo (GN), regula dos casos elementales de nuestro UC ( $\operatorname{los}$ casos 1 y 2$)$.

Los casos del UC son mutuamente excluyentes. Si un estado de cosas se encuadra en alguno de los casos del UC, no puede ser encuadrado en otro caso del UC.

Los casos del UC son conjuntamente exhaustivos. Cualquier estado de cosas del UD puede ser encuadrado en alguno de los casos del UC.

\section{EI ámbito normativo del sistema: El universo de soluciones}

En el ámbito normativo nos interesan las posibles soluciones para cada uno de los casos del UC. Nuestra pregunta es normativa; nos interesa el status normativo de ciertas acciones.

Una solución normativa puede ser expresada en términos de obligación, por ejemplo: «Es obligatorio que el nuevo poseedor abone los gastos necesarios», o en términos de derecho: «El anterior poseedor tiene derecho al abono de los gastos necesarios». Esto es lo que Kelsen denomina «derecho como correlato de una obligación activa» ${ }^{10}$. Es bueno advertir que no todos los derechos tienen como contrapartida una obligación activa, y tal es el caso del derecho de propiedad o el derecho a no pagar $^{11}$.

El derecho a realizar $\mathrm{u}$ omitir una acción será representado «Fp», donde «F» es el operador deóntico «Facultativo», $\mathrm{y}$ «p» la acción en cuestión.

El derecho a exigir que el otro realice una acción (la de pagar el gasto, por ejemplo) será representado $\langle\mathrm{F}(\mathrm{p}) »$, donde $\langle\mathrm{F} »$ es $« \mathrm{~F}$ acultativo», y $(\mathrm{p})$ la acción que debe realizar la contraparte.

La obligación de realizar una acción será representada «Op» donde «O» es el operador deóntico «Obligatorio» $\mathrm{y}$ «p» la acción en cuestión.

Tenemos por lo tanto tres soluciones normativas posibles:

Fp: derecho a hacer u omitir p.

$\mathrm{F}(\mathrm{p})$ : derecho a que la contraparte realice $\mathrm{u}$ omita $\mathrm{p}$.

Op: Obligación de hacer $\mathrm{p}^{12}$.

${ }^{9}$ Sobre la noción de caso complejo ver A \& B 1971, págs. 34 y 35.

${ }^{10}$ Kelsen 1981, págs. 138 a 143.

${ }^{11}$ Kelsen denomina a este tipo de derecho «derecho como correlato de una obligación pasiva». Kelsen 1981, págs. 143 a 145.

${ }^{12}$ En el modelo de A \& B 1971 todos los operadores deónticos pueden ser defi- 
En nuestro caso el legislador regula cuatro acciones distintas. Dos las regula en relación al nuevo poseedor (vencedor) y serán representadas del siguiente modo:

«p»: abonar el gasto o mejora.

«q»: abonar el mayor valor que adquiere la cosa principal en virtud de la mejora.

Otras dos acciones se refieren al anterior poseedor (vencido) y serán representadas del siguiente modo:

«r»: retener la cosa principal hasta que se le abone lo adeudado.

«s»: Ius tollendi.

El Ius tollendi: El ius tollendi es un régimen que el legislador establece para las mejoras suntuarias. Se divide entre mejoras separables y mejoras no separables de la cosa principal. Las no separables (una reforma de una casa, por ejemplo) carecen de derecho a reintegro. Las separables (una lámpara, por ejemplo) pueden ser retiradas por el anterior poseedor a menos que el nuevo poseedor quiera quedárselas, abonándolas. El abono puede ser del costo (p) o del valor del objeto al momento del traspaso (q).

\section{Reconstrucción del sistema normativo}

El problema que analizamos se halla regulado en el código civil español por los arts. 453 a 455, correspondientes al libro II, capítulo II «Efectos de la posesión».

Para la construcción de la matriz del sistema, debemos, en primer lugar, interpretar los artículos en cuestión, esto es, dotarlos de sentido. La interpretación de los textos legales es una cuestión teórica altamente debatida, y no es nuestra intención ahondar en este tema. Sin embargo es preciso señalar que se trata de una cuestión capital, ya que de la interpretación que se haga de los artículos dependerán las consecuencias normativas del sistema.

Es bueno señalar que los artículos que regulan nuestro UD no tienen grandes problemas interpretativos, ya que, como he afirmado en la introducción, su sentido puede precisarse dentro de la interpretación semántica.

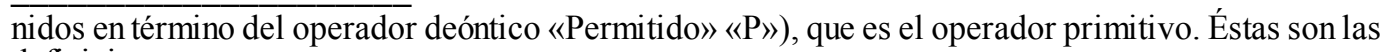
definiciones:

$$
\mathrm{Op}=\neg \mathrm{P} \neg \mathrm{p} \wedge \mathrm{Pp} ; \mathrm{Php}=\neg \mathrm{Pp} \wedge \mathrm{P} \neg \mathrm{p} ; \mathrm{Fp}=\mathrm{Pp} \wedge \mathrm{P} \neg \mathrm{P} \text {. Págs. 36, } 37 \text { y } 74 .
$$


Siguiendo a A \& B consideramos normas a los enunciados que correlacionan casos con soluciones. Por ejemplo, el art. 453 en su primera parte establece: «Los gastos necesarios se abonan a todo poseedor». Esto significa que si se da la circunstancia de tratarse de un gasto necesario, el nuevo poseedor debe abonarlo al anterior poseedor. Esta norma será representada del siguiente modo:

$$
\mathrm{GN}>\mathrm{Op}
$$

Esta norma puede leerse del siguiente modo: $\mathrm{Si}$ es gasto necesario (GN), entonces es obligatorio $(\mathrm{O})$ que el nuevo poseedor lo abone al anterior $(\mathrm{p})$.

Los arts 453 a 455 serán divididos en tantas normas como se pueda, todo dentro de la interpretación semántica:

Art. 453: Los gastos necesarios se abonan a todo poseedor; pero sólo el de buena fe podrá retener la cosa hasta que se le satisfagan.

Los gastos útiles se abonan al poseedor de buena fe con el mismo derecho de retención, pudiendo optar el que le hubiese vencido en la posesión por satisfacer el importe de los gastos, o por abonar el aumento de valor que por ellos haya adquirido la cosa.

Art. 454: Los gastos de puro lujo o mero recreo no son abonables al poseedor de buena fe; pero podrá llevarse los adornos con que hubiere embellecido la cosa principal si no sufriere deterioro, y si el sucesor en la posesión no prefiere abonar el importe de lo gastado.

Art. 455: El poseedor de mala fe abonará los frutos percibidos y los que el poseedor legítimo hubiera podido percibir, y sólo tendrá derecho a ser reintegrado de los gastos necesarios hechos para la conservación de la cosa. Los gastos hechos en mejoras de lujo y recreo no se abonarán al poseedor de mala fe; pero podrá éste llevarse los objetos en que esos gastos se hallan invertido, siempre que la cosa no sufra deterioro, y el poseedor legítimo no prefiera quedarse con ellos abonando el valor que tengan en el momento de entrar en la posesión.

El art. 453 ha sido dividido en cuatro normas. El primer párrafo determina la obligación del nuevo poseedor de pagar los gastos necesarios.

$$
\text { 453(1): (GN) > Op }
$$


La segunda parte del artículo establece derecho de retención, en favor del anterior poseedor de buena fe que haya realizado algún gasto necesario.

$$
\text { 453(2): }(\mathrm{GN} \wedge \mathrm{BF})>\mathrm{Fr}
$$

La tercera parte establece la obligación del nuevo poseedor de abonar los gastos útiles realizados por un anterior poseedor de buena fe. La norma otorga al poseedor dos alternativas sobre el modo de satisfacer dicha obligación: Abonar los gastos (p), o abonar el aumento de valor que halla adquirido la cosa principal en virtud del gasto o la mejora (q).

$$
\text { 453(3): }(\mathrm{GU} \wedge \mathrm{BF})>\mathrm{Op} \vee \mathrm{Oq}
$$

En la última parte se establece derecho de retención en favor del anterior poseedor de buena fe que haya realizado gastos o mejoras útiles.

$$
\text { 453(4): }(\mathrm{GU} \wedge \mathrm{BF})>\mathrm{Fr}
$$

El art. 454 regula el régimen de los gastos o mejoras suntuarias realizados por un anterior poseedor de buena fe. Los no separables no son de abono obligatorio, y los separables son retirables a menos que el nuevo poseedor prefiera abonar el importe del gasto. Esto es ius tollendi con derecho al abono del gasto.

$$
\text { 454(1): }(\mathrm{GS} \wedge \mathrm{BF})>\mathrm{Fs}(\mathrm{p})
$$

La primera parte del artículo 455 se refiere al régimen de la compensación de frutos. Como nuestro UD se refiere a las circunstancias en las cuales el nuevo poseedor debe (o no) abonar los gastos y mejoras realizados por el anterior poseedor, no nos interesa el régimen de los frutos. Por lo tanto este párrafo será excluido del presente análisis.

En la segunda parte del artículo, se establece el derecho del anterior poseedor de mala fe al abono de los gastos necesarios. El correlato de este derecho consiste en la obligación del nuevo poseedor de abonarle los gastos realizados.

$$
\text { 455(1): }(\mathrm{GN} \wedge \neg \mathrm{BF})>\mathrm{Op}
$$

La tercera parte del art. 455 establece el régimen de los gastos suntuarios realizados por un poseedor de mala fe. Se otorga el ius 
tollendi con derecho al abono del valor del objeto al momento del traspaso.

$$
\text { 455(2): }(\mathrm{GS} \wedge \neg \mathrm{BF})>\mathrm{Fs}(\mathrm{q})
$$

Estas siete normas conforman nuestro sistema normativo, que denominaremos S1. De ahora en más todas nuestras afirmaciones se referirán a este sistema $\mathrm{S} 1$, es decir al sistema conformado por los arts 453 a 455, tal y como han sido interpretados precedentemente.

\begin{tabular}{|c|c|c|c|}
\hline \multicolumn{4}{|c|}{ Gráfico 4} \\
\hline \multirow[t]{7}{*}{$\mathrm{S} 1=$} & 453(1): & $(\mathrm{GN})>\mathrm{Op}$ & \\
\hline & 453(2): & $(\mathrm{GN} \wedge \quad \mathrm{BF})$ & $\mathrm{Fr}$ \\
\hline & 453(3): & $(\mathrm{GN} \wedge \mathrm{BF})$ & $>\mathrm{Op} \vee$ \\
\hline & 453(4): & $(\mathrm{GN} \wedge \mathrm{BF})$ & $\mathrm{Fr}$ \\
\hline & 454(1): & $(\mathrm{GN} \wedge \mathrm{BF})$ & $F s(p)$ \\
\hline & 455(1): & $(\mathrm{GN} \wedge \neg \mathrm{BF})$ & $>\mathrm{Op}$ \\
\hline & 455(2): & $(\mathrm{GN} \wedge \neg \mathrm{BF})$ & $>\mathrm{Fs}(\mathrm{q})$ \\
\hline
\end{tabular}

Corresponde ahora establecer qué solución corresponde a cada caso elemental de nuestro UC. Veamos este procedimiento a través de un ejemplo:

La norma 453(1) establece la obligación de abonar (Op) toda vez que se trate de un gasto necesario (GN). De esta forma puede inferirse la solución Op para todos aquellos casos en que figure GN, que son los casos 1 y 2 de nuestro UC. Siguiendo este procedimiento la matriz del sistema $\mathrm{S} 1$ es la siguiente:

\section{Gráfico 5}

\begin{tabular}{|l|l|l|l|l|l|l|l|l|l|l|l|l|}
\hline & GN & GU & GS & BF & & $\begin{array}{c}453 \\
(1)\end{array}$ & $\begin{array}{c}453 \\
(2)\end{array}$ & $\begin{array}{c}453 \\
(3)\end{array}$ & $\begin{array}{c}453 \\
(4)\end{array}$ & $\begin{array}{c}454 \\
(1)\end{array}$ & $\begin{array}{c}455 \\
(1)\end{array}$ & $\begin{array}{c}455 \\
(2)\end{array}$ \\
\hline 1$)$ & + & - & - & + & $\mathrm{GS} \wedge$ & $\mathrm{Op}$ & $\mathrm{Fr}$ & & & & & \\
\hline 2$)$ & + & - & - & - & $\mathrm{GN}$ & $\mathrm{Op}$ & & & & & $\mathrm{Op}$ & \\
\hline 3$)$ & - & + & - & + & $\mathrm{GU} \wedge$ & & $\mathrm{Op \vee} \mathrm{Oq}$ & & $\mathrm{Fr}$ & & & \\
\hline 4$)$ & - & + & - & - & $\mathrm{GU}$ & & & & & & & \\
\hline 5$)$ & - & - & + & + & $\mathrm{GS} \wedge$ & & & & & $\mathrm{Fs}(\mathrm{p})$ & & \\
\hline 6$)$ & - & - & + & - & $\mathrm{GS} \wedge \neg \mathrm{BF}$ & & & & & & & $\mathrm{Fs}(\mathrm{p})$ \\
\hline
\end{tabular}


La matriz muestra que el sistema $\mathrm{S} 1$ es lógicamente consistente. Ningún caso elemental está correlacionado con soluciones normativas incompatibles entre sí, como sería la solución normativa $« \mathrm{Op} \wedge \mathrm{Fp} »^{13}$.

La matriz muestra que el sistema S1 presenta una redundancia en el caso 2, ya que tanto la norma 453(1) como la 455(1) le atribuyen la solución normativa «Op». Para tener un sistema independiente, esto es, equivalente en sus consecuencias pero más económico en su formulación, debería eliminarse alguna de las normas. En nuestro caso la norma 455(1) podría ser eliminada sin alterar las consecuencias normativas del sistema. No sucede lo mismo en relación a la norma 453(1), ya que su eliminación implicaría que el caso 1 se quedara sin solución normativa.

La matriz muestra, por último, que el sistema S1 presenta una laguna en el caso 4), esto es, la circunstancia en la cual un poseedor de mala fe ha realizado un gasto útil.

Esto no es lo mismo que afirmar que el código civil español tiene una laguna respecto del reintegro de los gastos y mejoras en la liquidación de situaciones posesorias. La matriz sólo permite afirmar que los arts. 453 a 455, interpretados de la forma en que han sido, constituyen un sistema normativo con una laguna.

Puede afirmarse que la laguna podría llenarse con alguna otra norma (y esto es lo que veremos en el próximo capítulo); pero se trataría de otro sistema, distinto de S1. Como afirman A \& B la completud (o incompletud) es un concepto relacional: Solo puede predicarse en relación a un UC, en relación a un US, y en relación a un sistema normativo, que en nuestro caso es S1. Como dicen los autores:

«Esto muestra la importancia de ponerse de acuerdo acerca de qué normas (es decir, acerca de qué sistema normativo) se está hablando cuando se afirma o niega la existencia de lagunas» ${ }^{14}$.

\section{CAPITULO SEGUNDO: MODIF ICACIÓN}

La tesis central del capítulo anterior consistió en afirmar la existencia de una laguna en el sistema $\mathrm{S} 1$.

Esto supone que si un juez se encuentra ante una situación en la cual un poseedor de mala fe ha realizado un gasto útil, el sistema $\mathrm{S} 1$ no le ofrece una solución normativa.

Como los jueces no pueden dejar de sentenciar, aún en los casos de ausencia de normas, corresponde modificar el sistema, esto es,

\footnotetext{
${ }^{13}$ Para la noción de contradicción normativa puede verse Alchourrón 1988.

${ }^{14}$ A \& B 1971, pág. 47.
} 
completarlo. Tenemos que inventar una norma para el caso 4 de nuestro UC.

Nos encontramos ante una tarea totalmente distinta de la emprendida en el capítulo primero. En aquel capítulo la tarea fue sistematizadora; esto es, analizar un conjunto de normas jurídicas y poner de manifiesto sus consecuencias lógicas. Dicha tarea fue descriptiva de un material jurídico (los arts. 453 a 455 del código civil español) previamente identificado mediante la interpretación semántica. La conclusión más importante de esa descripción fue la afirmación de que el sistema S1 era incompleto, por la existencia de una laguna en el caso 4.

Lo que haremos ahora no tiene ninguna relación con la descripción de un material jurídico. Ahora modificaremos el sistema, lo completaremos. No describiremos normas sino que las inventaremos. Podemos decir que nuestro objetivo es encontrar la norma más adecuada para completar el sistema.

Pero, ¿es posible el hallazgo de una norma tal que sea «la norma más adecuada»? ¿En base a qué parámetros es una norma «la más adecuada»?. Sin duda existen muchas respuestas a esta pregunta, respuestas dependientes de la teoría de la decisión judicial que se presuponga. Manuel Atienza ${ }^{15}$ señala tres tipos o clases de teorías de la decisión judicial.

Una teoría irracionalista de la decisión judicial, como lo es la de Alf Ross, sostiene que en aquellos casos en los que el derecho no ofrece una solución basada en las normas preexistentes, la decisión que se tome será esencialmente arbitraria, esto es, producto de la voluntad más que de la razón.

Una teoría hiperracionalista de la decisión judicial, como lo es la de Ronald Dworkin, sostiene que siempre existe una respuesta correcta para cada caso, contenida en el derecho preexistente; o bien en las normas jurídicas o bien en los principios jurídicos.

La teoría de la argumentación jurídica se encuentra a mitad de camino entre el irracionalismo y el hiperracionalismo ${ }^{16}$. La misma sostiene, que ante un caso difícil como lo es una laguna, es posible crear una norma que sea la más adecuada (o la más correcta), si se cumplen determinados requisitos ${ }^{17}$. Es importante advertir que la norma creada por medio de la argumentación jurídica no pertenece al material jurídico preexistente (como sostiene el hiperracionalismo), sino que puede crearse a partir de éste ${ }^{18}$. Por otra parte si se

${ }^{15}$ Atienza 1991, pág. 133.

${ }^{16}$ Cfr. MacCormick 1978, pág. 265; y Atienza 1991, pág. 133.

${ }^{17}$ Cfr. Alexy 1985, págs. 44 y 45.

${ }^{18}$ Esta afirmación no es del todo pacífica entre los teóricos de la argumentación. (Ver Atienza 1991, pág. 251). Esta cuestión será analizada en las conclusiones. 
cumplen con los requisitos de la argumentación jurídica, la decisión final puede ser calificada de «racional» ${ }^{19}$, a diferencia del irracionalismo que niega que puedan obtenerse resultados racionales.

Varios autores representan la teoría de la argumentación jurídica: La de Robert Alexy, la de Neil MacCormick, la de Aulis Aarnio, la de Aleksander Peczenick y, en nuestro país, la de Manuel Atienza. Para el análisis del caso concreto se usará como modelo la teoría de MacCormick.

MacCormick parte del carácter deductivo del razonamiento judicial, distinguiendo entre casos fáciles y casos difíciles. Los difíciles requieren una justificación mayor que los fáciles.

Un «caso difícil» puede plantearse al nivel de los casos genéricos o al de los casos individuales. Nuestro caso 4 es un caso genérico: todo gasto útil realizado por un poseedor de mala fe.

MacCormick señala varias causales para determinar si un caso es difícil. Nuestro caso 4 es difícil por problemas de determinación de la norma aplicable al caso genérico GU $\wedge \neg \mathrm{BF}$; causal que MacCormick denomina «problemas de relevancia ${ }^{20}$.

Esto nos permite afirmar que la lógica en general, y el modelo de A \& B en particular, es un instrumento importante para distinguir entre casos fáciles y difíciles tal como son las lagunas y las contradicciones en sistemas normativos.

Para la resolución de los casos difíciles se requiere una justificación de segundo nivel $^{21}$, que debe cumplir los requisitos de consistencia y coherencia en relación con el sistema jurídico en cuestión.

Una decisión es consistente cuando se basa en premisas normativas que no entran en contradicción con normas válidamente establecidas ${ }^{22}$.

Un sistema normativo es coherente si puede subsumirse bajo una serie de principios generales o valores que resulten aceptables, en el sentido de que configuren -tomados conjuntamente- una forma de vida satisfactoria ${ }^{23}$. Una decisión es coherente si la norma en que se basa es coherente en relación a los principios del sistema normativo en cuestión.

${ }^{19}$ Cfr. Alexy 1978, pág. 37.

${ }^{20}$ Cfr. MacCormick 1978, págs. 195/203-, y Atienza 1991, págs. 139/140.

${ }^{21}$ La distinción de MacCormick 1978 entre justificación de primero y segundo nivel es equiparable a la distinción de Alexy 1978 entre justificación interna y externa respectivamente.

${ }^{22}$ Sobre la contradicción entre normas jurídicas puede verse Alchourrón 1988.

${ }^{23}$ Esta formulación resulta objetable por su gran vaguedad. Como señala Atienza 1991 (pág. 145), puede considerarse a la coherencia como una justificación formalista y relativa. En este sentido puede tratarse de una justificación coherente con el sistema, pero incoherente desde una moral crítica o ideal. 
En base a estas dos tesis, consistencia y coherencia de los sistemas jurídicos, se intentará completar el sistema, esto es, inventar una norma para el caso 4 de nuestro UC: la norma más adecuada para completar el sistema debe ser consistente y coherente con S1. Por razones de comodidad, modificaré la descripción de la matriz del siguiente modo:

Gráfico 6

\begin{tabular}{|c|c|c|c|c|}
\hline & \multicolumn{2}{|r|}{$\mathrm{BF}$} & \multicolumn{2}{|r|}{$\neg \mathrm{BF}$} \\
\hline GN & 1 & $\mathrm{OP} \wedge \mathrm{Fr}$ & 2 & Op \\
\hline GU & 2 & $(\mathrm{Op} \vee \mathrm{Oq}) \wedge \mathrm{Fr}$ & 4 & \\
\hline GS & 5 & Fs (p) & 6 & Fs (q) \\
\hline
\end{tabular}

\section{La coherencia del sistema}

Corresponde como primer paso, determinar cuáles son los principios que rigen a S1. Esta tarea será realizada mediante la inducción ${ }^{24}$.

La inducción, de por sí, no es un proceso fiable. Sin embargo, en ciertos contextos, su resultado puede ser corroborado. Cuando se parte de un conjunto finito de casos (casos 1 a 6 de nuestra matriz) y se inducen los principios que los regulan, el resultado se corrobora cotejando si las normas se subsumen en dicho principio.

Ahora bien, que una inducción de principios sea corroborada no significa que dichos principios sean «realmente» los principios usados por el legislador; ni, mucho menos, que dichos principios pertenecen al sistema. La inducción que se realizará de los principios PA, PB, y PC es una de las tantas posibles, y, por lo tanto, puede darse que el sistema sea subsumible en otro conjunto de principios distinto del propuesto.

Un principio puede ser expresado como una regla, esto es un enunciado que correlaciona un caso con una solución ${ }^{25}$. Por ejemplo:

$$
\mathrm{PA}:(\neg \mathrm{GS} \wedge \mathrm{BF})>\mathrm{Fr}
$$

\footnotetext{
${ }^{24}$ Sobre la inducción jurídica puede verse A \& B 1971, pág. 130.

${ }^{25}$ Cfr. Atienza y Ruiz Manero 199 1. También A \& B 197 1, pág. 131 (la subsunción de casos elementales en casos complejos).
} 
Este principio estipula que los gastos no suntuarios de buena fe tiene derecho de retención. Corroborar este principio es comprobar si S1 lo cumple; y así es, ya que los casos 1 y 3 que se refieren a gastos no suntuarios de buena fe tienen la solución $\mathrm{Fr}^{26}$.

Este principio, que llamaremos «PA», no nos sirve para completar nuestra laguna (aunque servirá de premisa accesoria de un razonamiento). Necesitamos de principios más generales para completar el sistema.

Un principio también puede ser expresado a través de relaciones entre distintos supuesto ${ }^{27}$. Este camino seguiremos para inducir dos principios: uno en relación con el tipo de gasto y otro en relación con la buena o mala fe del anterior poseedor.

\section{En relación al tipo de gasto}

En cuanto a los gastos se establece el siguiente principio:

\section{PB: $\mathrm{DGN}>\mathrm{DGU}>\mathrm{DGS}$}

(el derecho de quien realizó un gasto necesario es mejor o igual que el de quien realizó un gasto útil; y éste mejor o igual que el de quien realizó un gasto suntuario).

¿Cuándo un poseedor tiene mejor derecho que otro poseedor? En algunas ocasiones se trata del derecho a un reintegro más oneroso. En otras ocasiones se trata del derecho accesorio de retención que algunos poseedores tienen y otros carecen.

La relación ser mejor o igual «>» es transitiva: Si x es mejor o igual que y, e y mejor o igual que $z$, entonces, $x$ es mejor o igual que $z$.

Corroboremos si PB se cumple en relación a los casos 1-3-5.

El derecho a percibir el costo (Op: caso 1) es mejor que el derecho a percibir el costo o el mayor valor ( $\mathrm{Op} \vee \mathrm{Oq}$ : caso 3) ya que al tener el deudor la alternativa entre $\mathrm{p}$ o q, siempre puede elegir la menos onerosa, que en general será el abono del mayor valor (q). A su vez los casos 1 y 3 tienen el mismo derecho de retención. El derecho de 1 en algunas ocasiones es mejor y en otras es igual que el de 3 .

El derecho a ([Op $\vee \mathrm{Oq}] \& \mathrm{Fr}$ : caso 3$)$ es mejor o igual que el ius tollendi (Fs[p]; caso 5). En cuanto a los bienes no separables es mejor, ya que 3 tiene derecho a reintegro y 5 carece de derecho a

\footnotetext{
${ }^{26}$ Los GN pueden ser definidos como: $\neg$ GU $\wedge \neg$ GS. Los GU pueden ser definidos como: $\neg \mathrm{GN} \wedge \neg$ GS.

${ }^{27}$ Esta técnica es la usada en Alchourrón 1961.
} 
reintegro alguno. En cuanto a los bienes separables el derecho es similar, ya que el ius tollendi es un derecho de retención sobre un objeto separable, ya que el anterior poseedor debe entregar el objeto si el nuevo poseedor decide abonárselo (Ver art. 454[1]).

En relación a los casos 2-4-6, sólo podemos cotejar el 2 con el 6, por la laguna del caso 4. El derecho a percibir el costo (Op: caso 2) es mejor o igual que el ius tollendi (Fs[q]: caso 6); por lo que cumplen con PB por la transitividad.

\section{En relación a la buena o mala fe}

En cuanto a la fe del anterior poseedor, siguiendo el mismo procedimiento, podemos establecer el siguiente principio:

$\mathrm{PC}: \mathrm{D} \mathrm{BF}>\mathrm{D} \neg \mathrm{BF}$

El derecho del anterior poseedor de buena fe (D BF), es mejor o igual que el derecho del anterior poseedor de mala fe $(\mathrm{D} \neg \mathrm{BF})$.

Para corroborar PC compararé el caso 1 con el 2 (GN), y el 5 con el 6 (GS). El caso 3 no se puede comparar con el 4 por la laguna de este último.

En cuanto a los GN, el caso 1 tiene un derecho de retención del que el caso 2 carece. Pero este último tiene derecho a un reintegro (p) similar a 1.

En cuanto a los GS, el caso 5 tiene el Fs(p), que es mejor o igual derecho que el Fs(q) del 6. En algunas ocasiones es mejor, tal como es el derecho al costo de la mejora (p), y en otras ocasiones es igual, como lo es en el caso de los bienes no separables.

Tenemos entonces los siguientes principios:

$\mathrm{PA}:(\neg \mathrm{GS} \wedge \mathrm{BF})>\mathrm{Fr}$

PB: $\mathrm{D}$ GN $>$ D GU $>$ D GS

$\mathrm{PC}: \mathrm{D} \mathrm{BF}>\mathrm{D} \neg \mathrm{BF}$

¿Constituyen estos principios una forma de vida satisfactoria? En algún sentido sí. Puede aceptarse que principios que premian la buena fe o la mejor utilidad del gasto, son principios satisfactorios.

La obtención de estos principios son un buen paso adelante. En base a PB y PC podemos dibujar el siguiente diagrama que representa la posición del caso 4) en comparación con el resto de los casos. 


\section{Gráfico 7}

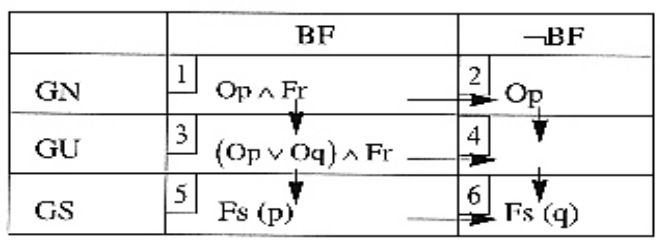

Las flechas verticales representan la coherencia del S1 en base al PB. Las flechas horizontales representan la coherencia del sistema en base al PC.

De la posición del caso 4 en el sistema pueden extraerse conclusiones importantes:

- Si la eventual norma N4 pretende ser coherente con S1, el derecho de 4 debe ser peor o igual que el de 3 en razón de PC. Debe ser peor o igual que el de 2 en razón de PB. Por la transitividad debe ser peor o igual que el de 1.

- Si N4 pretende ser coherente con S1, su derecho debe ser mejor o igual que el de 6 en razón $P B$.

En base a estas conclusiones intentaré establecer los límites de una decisión coherente con S1. Para ello crearé las normas N4 -coherente mínima- y N4 -coherente máxima-.

\section{La N4 coherente mínima}

N4 -mín- es el límite inferior de una decisión coherente.

La pregunta es la siguiente: ¿Cuál es la norma menos beneficiosa posible (para el anterior poseedor) dentro de los límites de la coherencia del sistema?

Para responder a este interrogante debemos comparar nuestro caso 4, con el 6. (Gráfico 7).

Lo primero que podemos afirmar es que una N4 -mín- tal como «Fp», esto es, la ausencia de derecho a reintegro, sería incoherente con el sistema, ya que PB establece que el caso 4 debe ser mejor o igual recompensado que el 6 , y en este supuesto sería peor recompensado.

Para establecer la N4 -mín-, podemos recurrir a una forma de argumento muy frecuentemente usado por los juristas; el argumento a fortiori, que constituye una clase de los argumentos a partir de 
principios. Estos argumentos pueden ser explicados a través de la lógica de relaciones ${ }^{28}$.

En nuestro caso, el argumento se basa en el carácter transitivo de la relación «ser mejor o igual que». Usando el caso 6 y el principio

$\mathrm{PB}$ el argumento tiene la siguiente estructura:

\section{Argumento 1)}

1) Quien realiza gastos sun-

1) DGS: Fs(q) (caso 6)

tuarios tiene el ius tollen-

di con derecho al reinte-

gro del mayor valor.

2) El derecho de quien realiza gastos útiles es mejor

2) $\mathrm{DGU}>\mathrm{DGS}(\mathrm{PB})$

o igual que el derecho de quien realiza gastos suntuarios.

3) Quien realiza gastos útiles tiene el ius tollendi

3) DGU: $[\mathrm{Fs}(\mathrm{q})] \vee[D>F s(q)]$

con derecho al reintegro

del mayor valor, o un derecho mejor.

4) De estas dos alternativas, «Ius tollendi con derecho al reintegro del mayor valor» es la menos beneficiosa para el anterior poseedor.

Analicemos el precedente argumento. La corrección de las premisas 1) y 2) es indispensable para la corrección de la conclusión 3).

La premisa 1) no resulta problemática; su corrección se debe a que es una norma que pertenece a $\mathrm{S} 1$, porque de él se deduce ${ }^{29}$.

No sucede lo mismo con la premisa 2). Esta premisa es el principio PB, que no se deduce de S1. Atacar la premisa 2) es atacar el principio PB.

${ }^{28}$ En Alchourrón 1961 se utiliza la lógica de relaciones. En Alexy 1978, en cambio, se utiliza la lógica de predicados de primer orden.

${ }^{29}$ Sobre el criterio de deducibilidad como criterio de pertenencia puede verse Caracciolo 1988, págs. 57 a 61 . 
El criterio de corrección del PB no lo otorga el criterio de deducibilidad sino la argumentación jurídica, concretamente, la tesis de la coherencia normativa de los sistemas jurídicos. La corrección de PB es más débil que la de la premisa 1). La corrección de 1) la otorga la lógica, y por ello su corrección es indubitada ${ }^{30}$, es decir, no abierta a contraargumentos. La corrección de 2), en cambio, es débil porque el principio PB no pertenece a $\mathrm{S} 1$ sino que fue creado a partir de él.

Los principios PA, PB y PC no son los únicos (lógicamente) posibles. Podrían proponerse otros principios para determinar la coherencia de S1. En este sentido la corrección de 2) está abierta a contraargumentos.

Sin embargo, creo que los principios PA, PB y PC pueden considerarse como adecuados porque cumplen los requisitos que MacCormick establece para la coherencia de los sistemas: Las normas de S1 se subsumen en PA, PB y PCi estos principios reflejan los criterios de reparto de derechos y obligaciones de $\mathrm{S} 1$, y, en este sentido, constituyen una forma de vida aceptable.

La conclusión 3) presenta una disyunción, con dos soluciones alternativas. Cualquiera de las dos se deduce de las premisas 1), 2) y 3). Las alternativas son Ius Tollendi con derecho al reintegro del mayor valor $[\mathrm{Fs}(\mathrm{q})]$, o un derecho mejor [D $>\mathrm{Fs}(\mathrm{q})]$.

Como nuestra hipótesis 4) es la norma menos beneficiosa dentro de los límites de la coherencia de $\mathrm{S} 1$, nos quedaremos con la primera alternativa. Por lo tanto:

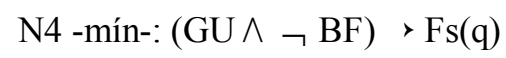

\section{La N4 coherente máxima}

N4 -máx- es el límite superior de la decisión. La pregunta es la siguiente: ¿Cuál es la norma más beneficiosa posible (para el anterior poseedor) dentro de los límites de la coherencia de S1? Para responder a este interrogante hemos de recurrir a los casos 1, 2 y 3 (gráfico 7).

En los casos 2-4 rige PB. El derecho de 4 ha de ser peor o igual que el de 2. Un argumento similar al argumento 1 sería el siguiente, utilizando el caso 2 y el principio PB:

${ }^{30}$ Indubitada si se aceptan los axiomas lógicos y la identificación de las normas realizada. 
Argumento 2)
1) DGN: Op
(Caso 2)
2) $\mathrm{DGN} \geq \mathrm{DGU}$
(PB)
3) DGU: $\left[O_{\mathrm{p}}\right] \vee\left[\mathrm{D}<\mathrm{Op}_{\mathrm{p}}\right]$
4) DGU: Op
(Caso 4 (-máx-)

Esta N4 -máx- establece para 4 un derecho igual que el del caso 2. Resulta, sin embargo, incoherente con el caso 3, en relación al principio PC. El caso 4 tendría derecho a un mejor reintegro (Op) que el caso $3(\mathrm{Op} \vee \mathrm{Oq})$, en contra del PC que establece que 3 ha de ser mejor o igual que 4.

Otra vía consiste en relacionar el caso 3 con el 4, donde rige PC. Éste sería el argumento:

\section{Argumento 3)}
1) DBF: $(\mathrm{Op} \wedge \mathrm{Oq}) \wedge \mathrm{Fr}$
(Caso 3)
2) $\mathrm{DBF} \geq \mathrm{D} \neg \mathrm{BF}$
(PC)
3) $\mathrm{D} \neg \mathrm{BF}:[(\mathrm{Op} \vee \mathrm{Oq}) \wedge \mathrm{Fr}] \vee[\mathrm{D}<(\mathrm{Op} \vee \mathrm{Oq}) \wedge \mathrm{Fr}](\mathrm{Caso} 4$
4) $\mathrm{D} \neg \mathrm{BF}:(\mathrm{Op} \vee \mathrm{Oq}) \wedge \mathrm{Fr}$
$(-$ máx-)

Esta N4 -máx- establece para 4 un derecho igual que el de 3. Sin embargo, esta norma resulta incoherente con el caso 2, en relación a PB: 4 tendría un derecho de retención del que 2 carece, en contra de PB que establece que el derecho de 2 ha de ser mejor o igual que el de 4.

De los argumentos 2 y 3 podemos concluir que la N4 -máx- ha de ser peor que los casos 2 y 3 (y que el 1 por transitividad) ya que si es igual a alguno de ellos, será mejor que el otro en algún aspecto (o en derecho de retención, o en derecho a un mejor reintegro).

Una tercera alternativa para establecer la N4 -máx- consiste en comparar conjuntamente los casos 1, 2, y 3 con el caso 4; usando los principios PB y PC. Para ello se requiere una especial preparación de las premisas.

El principio $\mathrm{PC}(\mathrm{DBF}>\mathrm{D} \neg \mathrm{BF})$ será disociado en dos principios, con la ayuda del principio PA que establece derecho de retención para los poseedores de buena fe. La disociación es la siguiente: 
$\mathrm{PC} 1: \mathrm{DBF}=\mathrm{D} \neg \mathrm{BF}$ (rige en cuanto al reintegro)

$\mathrm{PC} 2 \mathrm{DBF}>\mathrm{D} \neg \mathrm{BF}$ (rige en cuanto al derecho de retención)

El argumento sería el siguiente:

\section{Argumento 4}

A) -[PC1]- Si el igual derecho de 1 con 2 se concreta en el derecho a un reintegro igual (Op), entonces el igual derecho de 3 con 4 ha de concretarse en el derecho a un reintegro igual $(\mathrm{Op} \vee \mathrm{Oq})$.

B) -[PC2]- Si el mejor derecho de 1 sobre 2 se concreta en el derecho de retención, entonces el mejor derecho de 3 sobre 4 ha de concretarse en el derecho de retención.

Luego: 4 tiene derecho al reintegro (Op $\vee \mathrm{Oq})$. (A)

4 carece de derecho de retención.

La formalización de este argumento es mucho más compleja que la de los argumentos 1 a 3. Cada uno de ellos se basa en un caso y un principio; y este argumento se basa en tres casos y tres principios.

El argumento $4 \mathrm{~A}$, puede ser formalizado mediante los siguientes dos argumentos:

\section{A1}

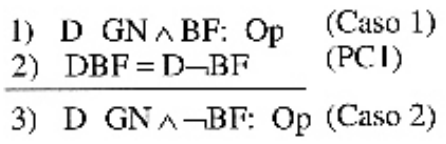

A2

$\begin{array}{ll}\text { 1) } \mathrm{D} G \mathrm{GU} \wedge \mathrm{BF}: \mathrm{Op} \vee \mathrm{Oq} & \text { (Caso 1) } \\ \text { 2) } \mathrm{DBF}=\mathrm{D} \neg \mathrm{BF} & \text { (PC1) }\end{array}$

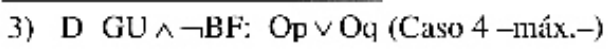

Estos dos argumentos comparan los casos 1, 2, 3 y 4 entre sí. A2 es el argumento decisivo: establece para el caso 4 un reintegro igual que el de 3 , igualdad basada en el argumento A1 que da el mismo reintegro a los casos 1 y 2 .

Si el legislador hubiera usado «realmente» el principio PC1, A1 es la explicación de porqué los casos 1 y 2 tienen derecho a un rein- 
tegro similar; y A2 es el fundamento de porqué el reintegro del caso 4 ha de ser similar al del caso 3.

La formalización del argumento $4 \mathrm{~B}$ es mucho más compleja que las aquí realizadas. Se requiere para ello el uso de una lógica de proposiciones normativas, ya que de lo que se trata es de afirmar que la norma (GU $\wedge \neg \mathrm{BF}>\mathrm{Fr})$ no está incluida en la norma N4 -máx-. $\mathrm{El}$ análisis de un tal argumento excede los objetivos del presente trabajo ${ }^{31}$.

La corrección de la conclusión: «4 tiene el derecho ( $\mathrm{Op} \vee \mathrm{Oq})$ y carece de derecho de retención» depende de la corrección de las premisas. Las premisas 1) de los argumentos no tienen problemas por que se deducen de S1. La corrección de las premisas 2) de los argumentos depende de que se acepte la disociación realizada del principio PC, en base a PA.

Esta N4 -máx- es coherente con S1, ya que respeta la posición del caso 4 con el resto de los casos. Es, por otra parte, la más beneficiosa (para el anterior poseedor) dentro de los límites de la coherencia de $\mathrm{S} 1$ : no es posible crear una norma que sea más beneficiosa (como la de los argumentos 2 y 3 ) y que a la vez sea coherente con S1. Por lo tanto:

$\mathrm{N} 4$-máx-: $(\mathrm{GU} \wedge \neg \mathrm{BF})>\mathrm{Op} \vee \mathrm{Oq}$

\section{Los límites de la argumentación en base a la coherencia}

Después de haber creado N4 -mín- y N4 -máx-, queda la duda de si es posible avanzar más, esto es, encontrar «la norma más adecuada». Creo que la respuesta a esta cuestión no puede ser sino negativa.

Esto podría desilusionar a quien pretende encontrar «la respuesta correcta». Creo, sin embargo, que esto es una de las características de la argumentación jurídica: en muchos casos difíciles no nos ofrece una respuesta correcta, sino sólo los límites de una decisión coherente. Pero no por ello deja de ser una herramienta útil: reconocer los límites de una herramienta no importa negar su utilidad, sino lo contrario.

Por tanto, la tesis de la coherencia normativa de los sistemas jurídicos nos permite afirmar lo siguiente: Cualquier decisión entre

${ }^{31}$ Una posible formalización del argumento B es la siguiente:
1) $《 \mathrm{GU} \wedge \mathrm{BF}: \mathrm{Fr} » \in \mathrm{Cn} \mathrm{S} 1$
(Caso 3)
2) $\quad \mathrm{DBF}>\mathrm{D}-\mathrm{BF}$
3) $(\mathrm{x}, \mathrm{y})[(\mathrm{BFX} \wedge \mathrm{BFy}) \wedge(\mathrm{X}>\mathrm{y})] \leftrightarrow[(« \mathrm{x}: \mathrm{Fr} » \in \mathrm{CnS} 1)>(« \mathrm{y}: \mathrm{Fr} » \neg \in \mathrm{CnS} 1)]$

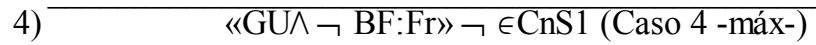


N4 -mín- y N4 -máx- (ambas incluidas) es una decisión coherente con S1.

Debe advertirse que N4 -mín- y N4 -máx- no son normas de S1: no pertenecen a S1 porque no se deducen de él. Son, más bien, una creación (del juez, de la ciencia jurídica) a partir de S1, en base a la tesis de la coherencia.

\section{La consistencia del sistema}

Tanto N4 -mín- como N4 -máx- son consistentes con S1, tomadas aisladamente. Ninguna de ellas es incompatible con alguna norma de S1.

Tomadas conjuntamente son, sin embargo, contradictorias entre sí. Se trata de una contradicción parcial-parcial ${ }^{32}$ en el caso de los gastos útiles no separables: según N4 -míncarecen de derecho a reintegro; según N4 -máx- tienen derecho al reintegro (Op $\vee \mathrm{Oq})$.

Esta contradicción resultaría problemática si se afirma que N4 -mín- y N4 -máxpertenecen a S1. Un juez se encontraría ante la obligación (jurídica) de aplicar ambas normas, por lo que debería incumplir alguna de las dos, ya que el cumplimiento de una implica (lógicamente) el incumplimiento de la otra ${ }^{33}$.

Si se afirma, a contrario, que dichas normas no pertenecen la cuestión no resulta problemática. Ambas normas constituyen los límites mínimos y máximos de una decisión coherente con S1. De esta forma el juez puede elegir cualquiera de las dos normas (o una intermedia), pero debe elegir sólo una de las normas posibles.

\section{CAPÍTULO TERCERO: ALGUNAS OPINIONES DE LOS JURISTAS}

En la dogmática civil española hay opiniones encontradas acerca de la solución normativa del caso 4 de nuestra matriz.

Pocos autores afirman explícitamente la existencia de la laguna; algunos la niegan. Resulta curioso constatar que todos coinciden en la descripción de los casos 1-2-3-5-6; pero difieren en la del caso 4.

Las discrepancias entre los autores se deben, básicamente, a que no se distingue con claridad la tarea descriptiva de la tarea reformu-

\footnotetext{
${ }^{32}$ Ross 1958, pág. 125.

${ }^{33}$ Esta afirmación parte de la base de que los jueces están obligados (jurídicamente) a aplicar las normas jurídicas en el acto de sentenciar. Cfr. Bulygin 1966. La relación cumplimiento-incumplimiento se basa en la tesis de Alchourrón 1988.
} 
ladora (o modificadora). Un buen ejemplo es la controversia entre quienes sostienen que le corresponde el ius tollendi similar al caso 6 (La Cruz Berdejo 1988, Albadalejo 1989) y quienes sostienen que el caso 4 carece de derecho a reintegro (Hernández Gil 1980, Delgado Echeverría 1975, Díez Picazo 1978).

La Cruz Berdejo sostiene:

«Nada dice el precepto de los gastos... (en) impensas útiles, de cuyo silencio cabría deducir que no son abonables las adiciones que puedan separarse. Aunque no lo diga el texto legal retirables sí lo son por aplicación «a maiore» de la regla sobre las impensas voluntuarias» ${ }^{34}$.

Hernández Gil, al contrario, sostiene:

«El poseedor de mala fe carece de derecho a retirar las mejoras útiles porque conforme a los arts 454 y 455, también carece de este derecho el poseedor de buena fe» ${ }^{35}$.

Si se trata de opiniones descriptivas del sistema, ambas resultan objetables: Del silencio de los artículos 453 a 455 no se deduce ninguna norma aplicable al caso 4. Por ello sólo puede afirmarse -metasistemáticamente- que el sistema tiene una laguna. Una afirmación que establezca una norma es -descriptivamente hablando- errónea.

Hernández Gil completa el sistema con la norma GU $\wedge \neg \mathrm{BF}>\mathrm{Fp}$ : El nuevo poseedor puede no pagar los gastos útiles de mala fe. Si se acepta que en S1 rigen los principios PA, PB y PC, esta propuesta es incoherente: el derecho de 4 es peor que el de 6, mientras PB establece que el de 4 debe ser mejor o igual que el de 6.

La Cruz Berdejo completa el sistema con la norma GU $\wedge \neg \mathrm{BF}>\mathrm{Fs}(\mathrm{q})$, un ius tollendi similar al del caso 6 . Esta propuesta coincide con nuestra N4 -mín-, y su adecuación depende de que se admita que el principio PB rige en S1. El argumento del autor sería similar a nuestro argumento 1), y PB actuaría como premisa implícita.

Esta propuesta es coherente con S1, pero parcial, ya que no es la única respuesta coherente con S1. Si un juez decide usar la N4 -máx-, su decisión también sería coherente con S1.

${ }^{34}$ La Cruz Berdejo 1988, pág. 118. En el mismo sentido Albadalejo 1989, pág. 120.

${ }^{35}$ Hernández Gil 1980. En el mismo sentido Delgado Echeverría 1975 y Díez Picazo 1978. 


\section{Conclusiones}

El presente trabajo partió de la idea de que la dogmática jurídica es una actividad compleja en la que pueden y deben distinguirse al menos tres etapas: 1) la identificación; 2) la sistematización; y 3 ) la reformulación (o modificación).

Se afirmó, además, que el éxito en enfrentar un caso difícil (como lo es una laguna) depende de distinguir claramente los distintos tipos de problemas y enfrentar cada uno con un método adecuado.

Siguiendo estos postulados se analizó un caso concreto (arts. 453 a 455 del código civil español) del siguiente modo:

1) Se identificaron las normas por medio de la interpretación semántica.

2) Se sistematizó el sistema con el método de A \& B 1971, y se detectó una laguna.

3) Se establecieron los límites mínimo y máximo de una decisión coherente y consistente con el sistema para completar la laguna. Esto último con el método de MacCormick 1978.

A partir de la tarea realizada pueden establecerse las siguientes conclusiones:

a) La interpretación del texto legal es una tarea importante, ya que de ella dependen todas las afirmaciones ulteriores. Algunas discrepancias entre los juristas (como lo es la existencia o no de una laguna) provienen de distintas interpretaciones del texto legal. Distintas interpretaciones importan distintos sistemas (distintas normas). Si se parte de sistemas distintos es razonable que se arriben a conclusiones distintas.

b) La lógica es una herramienta importante para la tarea sistematizadora. Ella nos pone de manifiesto dos propiedades formales de los sistemas normativos: la completud y la consistencia ${ }^{36}$.

c) (Consecuencia del anterior) La lógica sirve para detectar casos difíciles como lo son las lagunas (incompletud) y las contradicciones (inconsistencia).

d) En la tarea reformuladora, la lógica y la argumentación jurídica se complementan. Esto, sin embargo, es contrario a lo que sostienen muchos teóricos de la argumentación jurídica. Atienza 1993 sostiene que «una inferencia jurídica puede ser válida aunque sea menos que deductiva». Perelman $1960^{37}$ sostiene que la lógica

\footnotetext{
${ }^{36}$ A \& B 1971, pág. 100.

${ }^{37}$ Perelman 1960: Conferencia en la Universidad de Buenos Aires. Ver A \& B 1991, pág.
} XIX. 
(formal deductiva) es incapaz de dar cuenta de los argumentos a pari, a fortiori y a contrario: se requiere de una «nueva lógica» para justificar estos razonamientos.

Como se ha demostrado en la creación de N4 -mín- y N4 -máx- la lógica de relaciones es un buen instrumento para justificar este tipo de argumentos.

e) La teoría de la argumentación jurídica no siempre ofrece una única respuesta correcta (aunque esto no significa que a veces la ofrezca). En algunos casos, como el nuestro, sólo nos ofrece un «abanico» de respuestas coherentes con el sistema; un conjunto de respuestas correctas alternativas.

f) La distinción entre la tarea descriptiva y la reformuladora es una distinción crucial, que no siempre es tenida en cuenta. Las normas creadas en base a la argumentación jurídica no pertenecen al sistema en cuestión, y esto no ha sido advertido por muchos teóricos de la argumentación jurídica, que sostienen que el derecho positivo proporciona siempre, cuando menos, una respuesta correcta ${ }^{38}$.

g) Por último, y a modo de tesis final: Si, como afirman A \& B $1991^{39}$, «el valor de lógica consiste precisamente en que obliga a explicitar las premisas tácitas de los argumentos», entonces, el valor de la argumentación jurídica consiste en que permite justificar la corrección de tales premisas tácitas.

\section{BIBLIOGRAFÍA}

A \& B 1971: Carlos Alchourrón y Eugenio Bulygin, Normative Systems. Versión castellana de los autores titulada Introducción a la metodoloqía de las ciencias iurídicas y sociales. Editorial Astrea. Buenos Aires, 1975. (Las citas corresponden a la versión castellana).

A \& B 1991: Carlos Alchourrón y Eugenio Bulygin, Análisis lógico y derecho. Colección el derecho y la justicia, Centro de Estudios Constitucionales. Madrid, 1991.

Albadalejo 1989: Manuel Albadalejo, Derecho Civil III: Derecho de bienes. Volumen primero. Sexta Edición. Librería Bosch (Ed.). Barcelona, 1989.

Alchourrón 1961: Carlos Alchourrón, «Los argumentos jurídicos a fortiori y a pari». En Análisis lógico y Derecho, A \& B 1991.

Alchourrón 1986, «Systematization and change in the Science of Law». En T. Eckhoff, L.M. Friedman y J. Uusitalo (Eds.), Vermorft und Erfahunq in Rechtsdeuken des Geaenwart. Rechtstheorie 10. Berlín; Durcker \& Humblot, 171-184.

${ }^{38}$ Atienza 1991, pág. 251.

${ }^{39}$ A \& B 1991, pág. XIX. 
Alchourrón 1988: Carlos Alchourrón, «Conflicts of Norms and Revision of Normative Systems». Traducción castellana de Eugenio Bulygin titulada: «Conflictos de normas y revisión de los sistemas normativos. En Análisis lógico y Derecho A \& B 1991. (Las citas corresponden a la versión castellana)

Alexy 1978: Robert Alexy, Theorie der Juristischen Argumentation Traducción al castellano de Manuel Atienza e Isabel Espejo, titulada Teoría de la Argumentación Jurídica. Colección el derecho y la justicia; Centro de Estudios Constitucionales (Ed). Madrid, 1989. (Las citas corresponden a la versión castellana).

Alexy 1985: Robert Alexy: «Die Idee einer prozeduralen Theorie der Juristischen Argumentation». Traducción castellana de Ernesto Garzón Valdéz, titulada «La idea de una teoría procesal de la argumentaciónjurídica». En Derecho y Filosofía de Ernesto Garzón Valdés (Compilador). Editorial Alfa. Barcelona, 1985. (Las citas corresponden a la versión castellana).

Atienza 1991: Manuel Atienza, Las razones del Derecho: Teorías de la argumentación jurídica. Colección cuadernos y debates. Centro de Estudios Constitucionales. Madrid, 1991.

Atienza 1993: Manuel Atienza, «Lógica y Argumentación jurídica». (Respuesta a Moreso, Navarro y Redondo 1992). En vía de publicación en Doxa $N^{\circ}{ }^{11}$. Departamento de Filosofía del derecho, Universidad de Alicante (Ed.). Alicante.

Atienza y Ruiz Manero 1991: Manuel Atienza y Juan Ruiz Manero, «Sobre principios y reglas». En Doxa $N^{\circ}{ }^{\circ}$ 10. Departamento de Filosofía del derecho, Universidad de Alicante (Ed.). Alicante, 1991.

Bulygin 1966: Eugenio Bulygin, «Sentencia judicial y creación de derecho». En Análisis lógico y Derecho A \& B 1991.

Bulygin 1986: Eugenio Bulygin, «Dogmática jurídica y sistematización del derecho». En Análisis lógico y Derecho A \& B 1991.

Caracciolo 1988: Ricardo Caracciolo, Sistema Jurídico. Problemas actuales. Colección Cuadernos y Debates. Centro de Estudios Constitucionales (Ed.) Madrid, 1988.

Caracciolo 1993: Ricardo Caracciolo, «El concepto de "laguna": Descripción o Prescripción». En La noción de sistema en la Teoría del Derecho, del mismo autor. Ediciones Fontamara. México (en prensa).

Delgado Echeverría 1975: Jesús Delgado Echeverría, «Adquisición y restitución de frutos por el poseedor». En Anuario de Derecho Civil, tomo XXVIII. Publicación del Instituto Nacional de Estudios Jurídicos. Madrid, 1975.

Díez Picazo 1978: Luis Díez Picazo, Fundamentos del derecho Civil Patrimonial. Volumen segundo. Editorial Tecnos. Madrid, 1978.

Hernández Gil 1980: Antonio Hernández Gil, La Posesión. Editorial Civitas. Madrid, 1980.

Kelsen 1981: Hans Kelsen, Reine Rechtslehre Traducción de la segunda versión en alemán por Roberto J. Vernengo, titulada Teoría Pura del Derecho. Universidad Autónoma de México. México 1981. 
La Cruz Berdejo 1988: José Luis La Cruz Berdejo, Derechos reales:_Posesión y propiedad. José María Bosch Editor. Barcelona, 1988.

MacCormick 1978: Neil MacCormick: Legal Reasoning and Legal Theory. Clarendon Press. Oxford 1978.

Moreso, Navarro y Redondo 1992: José Juan Moreso, Pablo Eugenio Navarro y Cristina Redondo: «Argumentación Jurídica, Lógica y Decisión Judicial». En Doxa N. ${ }^{\circ} 11$. Departamento de Filosofia del Derecho, Universidad de Alicante (Ed.) Alicante, 1992.

1958: Alf Ross, On Law and Justice. Traducción castellana de Genaro Carrió, titulada Sobre el Derecho y la Justicia. Editorial Universitaria Buenos Aires (Eudeba), Buenos Aires, 1963. (Las citas corresponden a la versión castellana). 


\section{TABLA DE SÍMBOLOS}

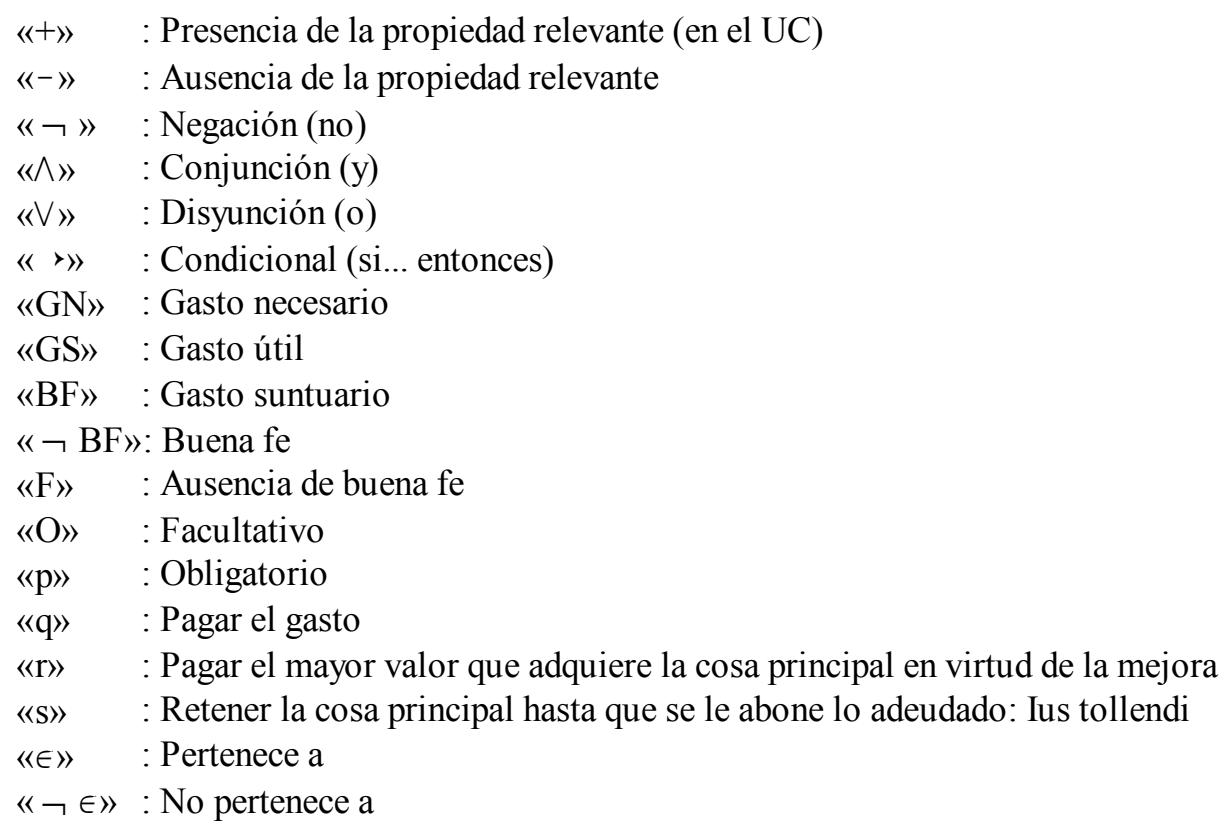



DOXA 17-18 (1995) 